\title{
Novel Membrane Materials for Reverse Osmosis Desalination
}

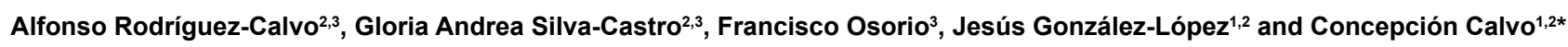 \\ ${ }^{1}$ Department of Microbiology, University of Granada, Spain \\ 2Institute of Water Research, University of Granada, Spain \\ ${ }^{3}$ Department of Civil Engineering, University of Granada, Spain
}

\begin{abstract}
The use of solvents in desalination processes limits the use of certain materials in the construction of the reverse osmosis membranes. Therefore now the research effort has focused on improving new materials. In particular, nanostructured materials will probably form the basis for new reverse osmosis membrane materials. This study makes a review of the new materials used for the construction of reverse osmosis membranes, highlighting the advantages and disadvantages that each of them gives to the desalination process. Firstly inorganic membranes, made mainly from zeolites, offer higher tolerance to a variety of feed waters and harsh cleaning methods. Secondly, two carbonderived materials as carbon nanotubes, exhibit high permeability and high rejection rate, and graphene, with high breaking strength and impermeability to molecules as small standard gases. Finally, a novel concept of membranes called Mixed Matrix Membrane (MMM) which combines organic and inorganic material and the benefits of each one.
\end{abstract}

Keywords: Membranes; Reverse osmosis; Desalination; Nanomaterials

\section{Highlights}

- Nanotechnology has opened the way to incorporate nanomaterials into $\mathrm{RO}$ processes.

- Research efforts are focussed on developing new materials that were less vulnerable to fouling and are easy to regenerate.

- Inorganic materials are potentially tolerant to waters which foul polymer membranes.

- CNT Membranes provide high water flux and rejection rate, and low energy consumption.

- Mixed matrix membrane has shown improved properties for numerous separation processes.

\section{Introduction}

Since the building of the first RO desalination plant, only polymeric membranes have been employed for industrial use. Thus, membrane modules have been improved by increasing membrane area per module. So it is appropriate review the historical development of commercially successful RO membrane materials and look forward to the novel nanostructured materials that will shape future trends in reverse osmosis membranes research. In fact, advances in nanotechnology have led to the development of nano-structured materials which may form the basis for new RO membranes. Thus, nanotechnology has opened the way to incorporate nanomaterials into RO processes [1].

Nowadays membranes technologies show advantages in both energy efficiency and high water quality. However, the use of organic membranes is highly limited due to the presence of solvents in the process of reverse osmosis for water desalination. Because of this, research efforts are focussed on developing new materials that were less vulnerable to fouling and were easy to regenerate [2].

Humplik et al. [3] affirmed that advances in nanotechnology have enabled unprecedented control on the fabrication of nanostructured materials, and in particular, makes possible to create well-defined, sizeselective, nanostructured filtration membranes.

Finally, different authors have introduced the concept of Mixed
Matrix Membrane (MMM), which combines organic and inorganic material.

\section{Inorganic Membranes}

\section{Zeolites}

Nowadays, inorganic membranes can be composed of a wide range of materials (from alpha alumina to zircon). The most common membranes are made of $\mathrm{Al}, \mathrm{Si}$, Ti or $\mathrm{Zr}$ oxides. Each oxide has a different surface charge in solution. Other membranes can be composed of mixed oxides of two of the previous elements, or are established by some additional compounds present in minor concentration. Their use is currently limited to applications where polymeric membranes cannot be used due to the high manufacturing cost. The structure of inorganic membrane modules is a flat membrane made up of a macroporous supporting layer and a meso or micro-porous active layer. Figure 1 shows the sub-nm inter-crystalline pores within the zeolite structure that allow the passage of water molecules and rejects the salt [1]. Currently, inorganic membranes are widely used in microand ultra-filtration applications whereas inorganic membranes for nanofiltration are under development [1].

Inorganic materials such as zeolites are capable of providing the required desalination properties while being potentially tolerant to waters which readily foul polymer membranes and/or can withstand more cost effective cleaning methods [4].

A group of researchers from the New Mexico Institute of Mining and Technology [5] reported the early results on the use of inorganic

*Corresponding author: Concepción Calvo, Institute of Water Research, Department of Microbiology, University of Granada, Spain, Tel: 34 958243093; E-mail: ccalvo@ugr.es

Received February 21, 2014; Accepted March 21, 2014; Published March 24 2014

Citation: Rodríguez-Calvo A, Silva-Castro GA, Osorio F, González-López J, Calvo C (2014) Novel Membrane Materials for Reverse Osmosis Desalination. Hydrol Current Res 5: 167. doi:10.4172/2157-7587.1000167

Copyright: () 2014 Rodríguez-Calvo A, et al. This is an open-access article distributed under the terms of the Creative Commons Attribution License, which permits unrestricted use, distribution, and reproduction in any medium, provided the original author and source are credited. 


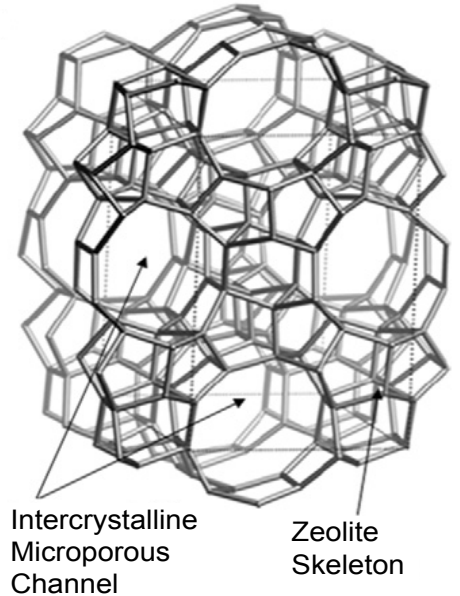

Figure 1: Micro-porous ceramic membrane structure: micro-porous channel in the crystalline structure MFI Zeolite [1].

membranes for RO desalination. Based on a molecular dynamic simulation which showed the feasibility (100\% of ion rejection) of using ZK-4 zeolites as membranes to separate water from aqueous $\mathrm{NaCl}$ solutions by Lin and Murad [6], this group investigated the RO separation mechanism and the feasibility of application of inorganic membranes.

Zeolites are crystalline aluminosilicate materials with uniform subnanometer- or nanometer-scale pores; microstructure is composed of 3-8 Å pores. Zeolite crystals occur naturally or can be synthesized in a laboratory environment using a high temperature furnace and an autoclave. In fact, varying synthesis temperature and time, crystal sizes can be controlled from a few nanometers to centimetres. Properties such as adsorption characteristics, geometry, ion exchange capabilities, and catalytic behaviour differ among the zeolite crystal families and can be tailored for a specific application by using the correct composition. Besides, due to the inert property of aluminosilicate crystal, zeolite membranes have superior thermal and chemical stabilities [3,7].

Theoretical calculations showed that zeolite membranes with pore sizes smaller than hydrated ion size could completely exclude them. The first experimental attempt was developed by Li et al. [8], who used hydrothermal synthesis to develop 0.5-3 $\mu \mathrm{m}$ thick membranes consisting of hydrophobic MFI (mordenite framework inverted) type zeolites with an average pore diameter of $5.6 \AA$ on a porous $\alpha$-alumina support. Under an applied pressure of $2.07 \mathrm{MPa}$ (20.7 bar) and with $0.1 \mathrm{M} \mathrm{NaCl}$ feedwater, the membranes rejected $76 \% \mathrm{of} \mathrm{Na}^{+}$ions while permitting a water flux of $0.112 \mathrm{~kg} \mathrm{~m}^{-2} \mathrm{~h}^{-1}\left(\sim 0.11 \mathrm{l} \mathrm{m}^{-2} \mathrm{~h}^{-1}\right)$. This lower rejection was attributed to ion transporting across nanometer-sized interstitial defects created during the membrane synthesis process [3]. Lee et al. [1] exposed that in a test using a feed containing mixed ion species the rejection of bivalent cations was higher than for monovalent ions, or, in other terms, the rejection of sodium ions in a mixed ion solution was lower than for a pure solution of $\mathrm{NaCl}$. These results show that the filtration mechanism is not only dependent on size exclusion, but also on Donnan exclusion due to the charged double layer induced by adsorbed ions on the pore or the intercrystalline walls. Li et al. [9] stated that the salt rejection depended on the formation and size of EDLs (electric double layer) at the surface of the intercrystalline defects. The thickness of this layer decreases with an increase in ion concentration; in low salt concentrations, the EDLs can overlap and prohibit the transport of salt ions, while, in high salt concentrations, EDLs become thinner and no longer overlap, allowing ions to pass through the intercrystalline defects. All these experimental results showed that nanometer-sized intercrystalline defects controlled the majority of ion transport and represent a challenge for the fabrication of zeolite membranes $[1,2,3,8,10]$. In fact, Dong et al. [10] stated that polycrystalline zeolite membranes inevitably contain nanometerscale intercrystalline pores, which decrease the efficiency of ion rejection especially for high concentration feed solutions and solutions containing multivalent cations.

Recently Li and Wang [11] stated that typical zeolite membranes are prepared by the hydrothermal deposition method on suitable porous inorganic or stainless steel supports. A supporting material must provide the physical durability to membranes, but none effect on fluid flow and salt rejection than the zeolite separation layer.

Two basic parameters, salt rejection and water flux, were too low to be of practical use, so subsequent work has been conducted to improve both by modifying the zeolite structure [1]. The Si / Al ratio was optimized to improve them. The $\mathrm{Al}$ content in the membrane can alter the surface hydrophilicity and therefore affinity with water. Besides, Defects in the crystal structure are minimized by secondary growth of a zeolite layer on zeolite seeded onto a porous $\alpha$-alumina substrate [4]. This combined effort generated a remarkable improvement, with a $2 \mu \mathrm{m}$ thick zeolite membrane with 50:50 Si/Al ratios. The water flux increased from 0.112 to $1.129 \mathrm{~kg} \mathrm{~m}^{-2} \mathrm{~h}^{-1}$ and ion rejection increased from $90.6 \%$ to $92.9 \%$ as $2.0 \mathrm{~mol} \% \mathrm{Si}^{4+}$ in zeolite framework was substituted by $\mathrm{Al}^{3+}$ [12]. In recent reports from the same group, the thickness of the membrane has been further reduced to $0.7 \mu \mathrm{m}$, providing excellent organic (>99\%) and salt rejection $(97.3 \%)$ as well as nearly 4 times improvement in water flux $[1,13,14]$.

Similar to the polymeric membranes, performance of zeolite membranes may be affected by transmembrane pressure, temperature, ion concentration and composition of the feed salt solution. An increasing of feed temperature has a greater influence on the ion permeation than on the water permeation, resulting in a decline of the ion rejection. On the contrary, increasing the transmembrane pressure can enhance both the water flux and ion rejection rates because the ion flux is much less affected by pressure compared to the water flux. These findings suggest that for RO on zeolite membranes, which possess excellent thermal and mechanical stabilities, operating at elevated temperatures and high hydraulic pressures is desirable for enhancing the separation efficiency [15]. Increasing feed ion concentration results in an exponential increase of ion flux because both the driving force for ion transport and effective pore size increases with increase of ion concentration due to diminishing of the electric double layer at increased ion concentration $[9,11]$.

Thus, zeolite membranes have shown higher permeability and selectivity than polymeric membranes. They exhibit better resistance to harsh chemical conditions and can withstand high pressure and temperature, which constitute potential advantages for desalination [11].

Finally Lee et al. [1] stated that though the improvement of zeolite membranes has been remarkable in the past 10 years, their performance and economics are still no match for polymeric membranes. The zeolite membrane thickness is still at least 3 times higher than the current state of the art polymeric $\mathrm{RO}$ membranes, causing higher resistance to water flux. As a result, inorganic membranes require at least 50 times higher membrane area than polymeric ones to achieve an equivalent production capacity. 


\section{Carbon nanotubes membranes}

To overcome the limits of current polymeric membranes, which suffer from a trade-off between salt rejection rate and permeability, new types of membrane with higher permeability and rejection rate have been developed. These membranes use carbon nanotubes (CNTs) as membrane pores [16]. CNTs are an allotrope of carbon consisting of rolled-up sheets of graphitic layers [3]. Ahn et al. [17] stated that the cylindrical shape of single walled nanotubes (SWNTs) can be imagined virtually by wrapping them in a layer of graphite called graphene.

These CNT membranes could potentially provide a solution to water shortages, as they seem to outperform existing membranes by providing higher water flux and lower energy consumption, so they could be a dramatic improvement over the conventional membranes. In contrast, the feasibility of CNT membranes has not fully investigated, as they are still in the laboratory stage of development and not yet commercially available. Fabrication of CNT membranes, which have controlled geometry, porosity, and pore shapes, is also challenging $[16,18]$.

According to Ahn et al. [17], CNTs can be classified into two categories according to the fabrication methods; (1) vertically aligned CNT membranes (VA-CNT membranes), and (2) mixed (composite) CNT membranes. With respect to VA-CNT membranes, nanotubes are arranged straight up and perpendicular to the membrane surface, and bound to each other by an organic or inorganic filler material. The authors highlight that an important advantage of VA-CNT membranes is that water flux would be very rapid due to the short nano-channel length and compactness of the nanotube forest. The features of VACNT are the following:

- CNTs are aligned vertically.

- CNTs' forest is compacted densely.

- Water flux is supposed to be fast drastically.

- Functional group can be attached at the tip of CNTs or on the membrane surface conveniently.

- Fabrication procedures are complicated.

- May need specially adjusted operating system [17].

In high yield synthesis techniques, the majority of the nanotubes adopt a metallic armchair conformation [19], though difficulties in purifying for monochiral nanotubes constrain commercial and environmental applications of SWNTs. Double-walled and multiwalled nanotubes (MWNTs) are the one-dimensional analogues of carbon onions. MWNTs share many characteristics of bulk SWNTs since coupling across the $0.34 \mathrm{~nm}$ interlayer distance of MWNTs is weak. MWNTs do not exhibit the metallic properties of SWNTs, instead displaying the semiconducting characteristics of bulk graphite [20]. A sketch of SWNT and MWNT is shown in Figure 2.

The way graphene winds can be described by a pair of indices (n, $\mathrm{m}$ ). The indices $\mathrm{n}$ and $\mathrm{m}$ are integers indicating the number of unit vectors along two directions of graphene. The inner diameter of a nanotube can be calculated from the "rolled up" vector as follows [22].

$$
d_{\mathrm{in}}=\left(\frac{a}{\pi}\right) \sqrt{\left(n^{2}+m^{2}+n m\right)-2 \mathrm{r}_{c}}
$$

$\mathrm{d}_{\mathrm{in}}$, inner diameter (I.D.) of nanotubes; a, lattice parameter of graphene $(=2.46 \AA)$; and $r_{c}$, van der Waal's radius of a carbon atom (1.7 A) [17].
Advances in synthesis procedures, such as chemical vapour deposition (CVD), have recently enabled ultra small diameter SWNT and lengths up to a few centimetres [23]. Controlled synthesis techniques have led to the recent discovery of high water fluxes through CNT membranes $[16,24]$, opening the possibility of using such CNT membranes for RO $[3,25]$.

Nano-pores should be homogeneously distributed on the surface layer to enhance the performance of CNT membranes. Above all, it is crucial to produce high quality CNTs. Nanotubes should be prepared uniformly to obtain high desalination capacity. Thus, homogeneity of nano-pores is crucial, and the inner diameter of nanotubes should be distributed within a very narrow range. The inner diameter of MWNTs can range from a few nanometers to tens of nanometers, which may result in poor desalination capacity. In contrast, SWNTs inner diameters are $<1-2 \mathrm{~nm}$, but their manufacturing procedure is relatively complicated. During the manufacture of nanotubes, the dependence of carbon nanotube inner diameter and number of walls upon the size of the inorganic catalyst has been studied [17,26,27].

Results obtained by different authors using the MD modelling and performing experiments showed that water fluxes several magnitudes greater than those predicted by continuum hydrodynamics through CNTs. In the same way, mechanistic insights into the ultrafast water transport have been provided by MD simulations [16,24,28,29]. Kolesnikov et al. [30] suggests that interactions of water molecules with the CNT wall are lower than the interactions between the water molecules. This suggestion was proved by confinement of water molecules in a CNT, leads to the formation of an 'ice shell plus water chain structure". The results obtained by the study of the transport of water molecules through CNTs with varying degrees of hydrophobicity and surface roughness, showed that for fast water transport are necessary both hydrophobicity and the atomistic smoothness of CNT walls, resulting in nearly frictionless flow [28].

More recently, Falk et al. [31] demonstrated that the curvature modulates the interaction energy landscape of water molecules with the CNT such that the friction decreases with decreasing CNT diameters, and vanishes below a CNT diameter of $0.5 \mathrm{~nm}$; these results were taken using MD simulation. Likewise, the simulations indicated that the friction was higher for water molecules interacting with the outer surface of the CNTs, concluded that the friction coefficient between the water molecules and the CNT wall is highly dependent upon the curvature of the graphitic surface of the CNTs [30]. Majumder et al. [16] and Holt et al. [24], who experimentally verified fast mass transport, made CNT membranes by filling the interstitial space of an array of vertically

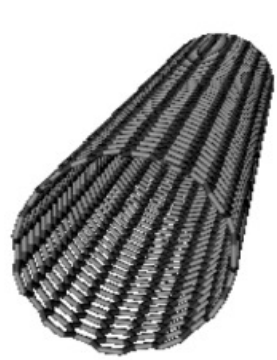

SWNT

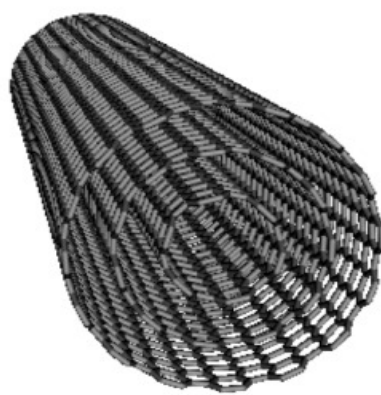

MWNT
Figure 2: Molecular structure of SWNT and MWNT [21] 
aligned CNTs with polystyrene and silicon nitride, respectively. They measured the flow rate of water, ethanol, isopropanol, hexane, and decane through CNTs under 1 bar of pressure. It showed that, firstly, increasing fluid viscosity did not decrease liquid flux, and secondly, the flux predicted by continuum hydrodynamics was $4-5$ orders of magnitude smaller than the liquid flux through the membrane.

Hinds's research group introduced the first prototype for a VACNT and found that water flow rates increased 4- to 5-fold over those of conventional fluid flow, which was estimated from the HagenPoiseuille equation, by performing a series of pressure-driven flow experiments. However, they did not report ion selectivity, which is strongly related to desalination potential during the desalination process [32]. In addition, Holt et al. [24] improved upon size selectivity in a membrane of aligned double-walled CNTs with an inner diameter less than $2 \mathrm{~nm}$, while maintaining high mass transport rates.

As nanotubes are hydrophobic, there is a decreasing water flux due to the movement of water molecules hindered in areas adjacent to the surface of a CNT membrane. However, the hydrophobic interior wall is favourable, so it helps the water molecules to be transported at ultrahigh speed once water molecules enter the membrane. Therefore, it may be beneficial to functionalize the end tips of nanotubes to change hydrophobicity of the CNT membrane and eventually enhance ion selectivity. However, according to Ahn et al. [17], a further detailed study will be necessary to develop a coating with densely charged polymers over the entire membrane surface as well as CNT end tips to increase salt rejection efficiency. In the same way, Humplik et al. [3] reported that membranes comprising CNTs with chemically modified tips with charged groups were developed because CNT diameters were too large to act as molecular sieves for excluding salt ions even though the high flux through the CNT membranes were applied. Fornasiero et al. [25] functionalized with carboxyl groups the pore entrance of these CNT arrays, which successfully achieved of $40-60 \%$ salt rejection for dilute $(<0.01 \mathrm{M})$ salt concentrations of $\mathrm{KCl}$, and nearly $100 \%$ salt rejection for dilute solutions of $\mathrm{K}_{3} \mathrm{Fe}(\mathrm{CN})_{6}$. The higher rejection ratio for $\mathrm{K}_{3} \mathrm{Fe}(\mathrm{CN})_{6}$ may be attributed to the greater electrostatic repulsion between the trivalent anion and the carboxyl groups at the CNT entrance. The salt rejection was poor for higher salt concentrations that correspond to a smaller electrostatic Debye screening length.

Various studies have reported that CNTs trigger inactivation of bacterial cells by attacking cell walls. These membranes suppress biofilm formation on deposited surfaces; SWNTs are more often fatal to bacteria than MWNTs. Two trials have been conducted to reduce biofouling using nanotubes. Therefore, this low biofouling surface may reduce the maintenance required for commercialized $\mathrm{NF}$ and $\mathrm{RO}$ membranes $[17,33,34]$. The CNT membrane process will require less energy consumption than that of other membrane processes because it can run without high-pressure pump and due to the fact that the specific surface area of the membrane element attaining the targeted permeate volume should be diminished [17]. Commercially available SWNTs and MWNTs are replete with defects, metal catalyst contamination, and physical heterogeneities. While a number of purification schemes have been developed [35], variation between samples is a challenge for research on the implications and commercial applications of CNTs [20].

\section{Graphene}

Graphene is a carbon-based material considered as a potentially selective material for membranes. According to Humplik et al. [3] graphene is a single layer of graphite with atomistic thickness, consisting of a lattice of hexagonally arranged $\mathrm{sp}^{2}$-bonded atom, which exhibits a high breaking strength [36] and impermeability to molecules as small as standard gases including helium [37]. These properties suggest to create graphene ultrathin high flux membranes that can act as molecular sieves [3,38]. Furthermore, it has been developed the rollto-roll production of graphene on ultralarge copper, which indicates the feasibility of large-scale membrane fabrication [39].

Some recent studies have explored the transport of or ions through pores in graphene membranes [40,41]. Sint et al. [40] showed that graphene monolayers could serve as ionic sieves of high selectivity and transparency. More Recently, Suk and Aluru [41] explored the water transport through a porous graphene membrane and compared the results with water transport through thin (less than $10 \mathrm{~nm}$ in thickness/ length) carbon nanotube (CNT) membranes. For smaller diameter membranes, water flux was lower through the graphene membrane compared to that of the CNT membrane, but for larger diameter pores, water flux is higher through the graphene. These results suggest that graphene membrane can be used as an ultra efficient water transporter, compared to thin CNT membranes, whenever the diameter is larger than $0.8 \mathrm{~nm}$. However, Humplik et al. [3] stated that experimental measurements of water transport and salt rejection remain to be realized.

Cohen Tanugi and Grossman [38] showed that nanometer-scale pores in single-layer freestanding graphene can effectively filter $\mathrm{NaCl}$ salt from water. They stated that nanoporous membranes can allow for fast convective water flow across well-defined channels. In contrast with classical RO membranes, where water transports slowly via a solutiondiffusion process. Using classical molecular dynamics simulations, they examined differences in desalination dynamics related to pore size, pore chemistry, and hydrostatic pressure applied.

According their simulations, the permeability scales linearly with pore area, as expected from the Hagen Poiseuille equation. On the other hand, the effect of pore chemistry has no clear analog in macroscopic fluid dynamic. For a given pore size, water permeability is significantly enhanced by hydroxylation compared with the hydrogenated case. This behaviour is due to the fact that hydrophilic functional groups increase the water flux by allowing for a greater number of hydrogenbonding configurations inside the pore. On the other hand, salt rejection decrease with increasing pore size, which is expected from a size exclusion argument. However, the dependence of salt rejection on applied pressure is noteworthy; the salt rejection of a given pore decreases at higher applied pressures, which is the opposite of what is observed in diffusive RO membranes. They attributed this difference in behaviour to the large effective volume of ions in solution, which causes them to respond more sensitively to pressure increases than water molecules. This is in contrast with RO membranes, in which the governing driving force for salt passage is osmotic pressure and where water flux increases faster than salt flux with rising pressure. These results indicated that pore chemistry also has a notable effect on salt rejection. For a given pore size and applied pressure, the salt rejection is lower for hydroxylated pores. They attributed this effect to the fact that hydroxyl radical $(\mathrm{OH})$ functional groups can hydrogen-bond with salt ions much like water molecules do, which results in a lower free energy barrier to ionic passage [38].

Finally, their examination of the structure of water in the pore vicinity revealed that the hydrophobic character of hydrogenated pores reduces the water flow by imposing additional conformational order on the system, even as the limited hydrogen bonding allows for greater salt rejection relative to hydroxylated pores [38]. 


\section{Mixed matrix membranes}

Mixed matrix membrane (MMM), also known as composite membrane, produced by dispersing fillers in a polymeric matrix, has shown advantages in the development of advanced membranes with improved properties for numerous separation processes [11].

The concept of MMM, the combination of organic and inorganic material, is not new. In 1980 a silicalite-cellulose acetate MMM for gas separation was developed, showing superior selectivity to conventional polymeric membranes and in the 1990s water / ethanol separation via pervaporation. The incorporation of inorganic materials into organic RO TFC membranes only started in the early 2000s. The main objective of MMM is to combine the benefits offered by each material, i.e. the high packing density, good permselectivity, and long operational know-how of polymeric membranes, coupled with the superior chemical, biological and thermal stability of inorganic membranes [1].

\section{Nano-Particle/Polymeric Membranes}

According to Lee et al. [1], zeolite nano-particles have been used to prepare MMMs. Lind et al. [42] stated that the concept of a mixed-matrix membrane, where a small filler material is dispersed throughout a larger polymeric matrix, has brought new degrees of freedom to the development of advanced membrane materials for numerous separation processes. Jeong et al. [43] exposed that these NaA-type zeolite particles, which are characterized by being very hydrophilic, are in the size range of $50-150 \mathrm{~nm}$ with a $\mathrm{Si} / \mathrm{Al}$ ratio of 1.5. The zeolite nano-particles are dissolved into a cross-linking agent solution (trimesoyl chloride dissolved in hexane) before the interfacial polycondensation reaction takes place. A homogeneous dispersion of zeolite particles is achieved using ultrasonication before the standard interfacial polymerization is carried out. RO membranes with various zeolite loadings were prepared and consequent changes in membrane characteristics were observed, i.e. the membranes were smoother, more hydrophilic and more negatively charged with increasing nanoparticle loading. The MMM membrane exhibited $90 \%$ of flux and a slight improvement in salt rejection relative to the hand cast TFC membrane without zeolite nano-particles. Authors suggest that this could be a result of enhanced Donnan exclusion by the zeolite particles and changes of membrane morphology $[1,42,43]$.

Lind et al. [42] reported that the presence of zeolite nanoparticles may change polyamide film structure, which was likely induced by the formation of microporous defects and reduced the crosslinking extent. As a result, the zeolite A-polyamide TFN membranes exhibited improved permeability while retaining a good rejection.

The dramatic increase in solute permeability might limit the application of TFN (thin film nanocomposite) membranes used in traditional RO systems designed to operate at extremely low water fluxes. Moreover, these results offer additional support for the hypothesis that zeolite nanoparticles change bulk polyamide film structure possibly by creation of microporous defects as well as reduced cross-linking through heat released during the polymerization reaction [42].

On the other hand, Kim et al. [44] performed a trial where $\mathrm{TiO}_{2}$ nano-particles $(<10 \mathrm{~nm})$ were subsequently dip-coated onto an interfacial polymerized fully cross-linked polyamide TFC membrane with a surface layer functionalised with carboxylate groups. These carboxylate groups are necessary for the self-assembly of $\mathrm{TiO}_{2}$ within the barrier layer via an adsorption mechanism. Testing with E. colicontaining feed water has shown superior anti-biofouling properties, especially with the aid of UV excitation, without compromising the flux and salt rejection performance of the original membrane. No significant loss of $\mathrm{TiO}_{2}$ nano-particles from the membrane was observed after a continuous 7-day RO trial [1].

However, in another trial performed by Lee et al. [45], the addition of $\mathrm{TiO}_{2}$ during in situ interfacial polymerization of polyamide produced the TFN membranes with improved pure water flux and enhanced hydrophilicity. As $\mathrm{TiO}_{2}$ loading increased, salt rejection initially increased, accompanied with a slight change of flux; however, further increased $\mathrm{TiO}_{2}$ loading led to a reduction of salt rejection with a jump of water flux and poor mechanical strength. They believed that the interference of interfacial polymerization by inorganic $\mathrm{TiO}_{2}$ nanoparticles became significant at higher $\mathrm{TiO}_{2}$ loadings, resulting in a lower degree of polymerization.

Li and Wang [11] stated that the fabricated membranes having poor mechanical property may limit their practical application, therefore it is necessary to optimize the membrane fabrication and polymerization processes by considering the effect of inorganic fillers.

\section{Carbon Nano-Tube/Polymeric Membranes}

Anh et al. [17] described an earlier model of a mixed-CNT membrane which was mainly designed to upgrade a UF membrane with nanotubes. MWNTs (up to $5 \%$ by weight volume) were blended with polysulfone (PSf), and water fluxes were measured under an operating pressure of 1- 4 bar [46]. They reported that the MWNT/ PSf membrane revealed two pieces of conflicting data according to the molecular weight of the solute. For an aqueous solution of polyethyleneoxide 100,000 , the solute rejection efficiency was high $(>95 \%)$ and the water flux was measured at $14-17 \mathrm{l} \mathrm{m}^{2} \mathrm{~h}^{1}$. In contrast, the solute rejection efficiency was reduced by $20-60 \%$ for aqueous solution of poly-vinylpyrrolidone (PVP) 55,000, whereas the water flux was increased to $>40 \mathrm{l} \mathrm{m}^{2} \mathrm{~h}^{1}$. Choi et al. [46] postulated that the plugging effect between both sizes of nano-pores and the solute molecule might contribute to differences in the solute rejection efficiencies and, according to them, it seemed to be a dilemma to accomplish a higher permeability and rejection rate at the same time with the mixed-CNT membrane [17].

On the other hand, Anh et al. [17] reported that functionalized MWNTs blended with PSf have been prepared for UF membranes [47]. MWNTs were modified by attaching isocyanate and isophthaloyl chloride functional groups, and protein adsorption on the membrane surface was suppressed. Thus, it was anticipated that a functionalized MWNT/PSf membrane would alleviate membrane biofouling.

According to Lee et al. [1] the fabrication of the CNT/polymeric membrane in some experimental studies [16,27,32], involves multiple complex steps, such as catalytic growth of CNTs onto expensive substrates, polymer filling of the inter-tube spaces, substrate removal, and CNT tip opening via etching. Furthermore, they stated that the size distribution of the CNT diameters is still not small enough to complement the simulation studies performed.

Ratto et al. [48] realized a patent which could overcome this problem. They disclosed the blending of CNTs into solutions, preferably cross-linking agent solutions (trimesoyl of isophthalic chlorides), for formulation of composite polymeric membranes (Figure 3).

They assure that CNTs can be effectively embedded onto the barrier layer formed by conventional interfacial polymerization on a micro-porous polyethersulfone support. In this patent the CNTs are functionalized with octadecylamine; Lee et al. [1] reported that CNTs 


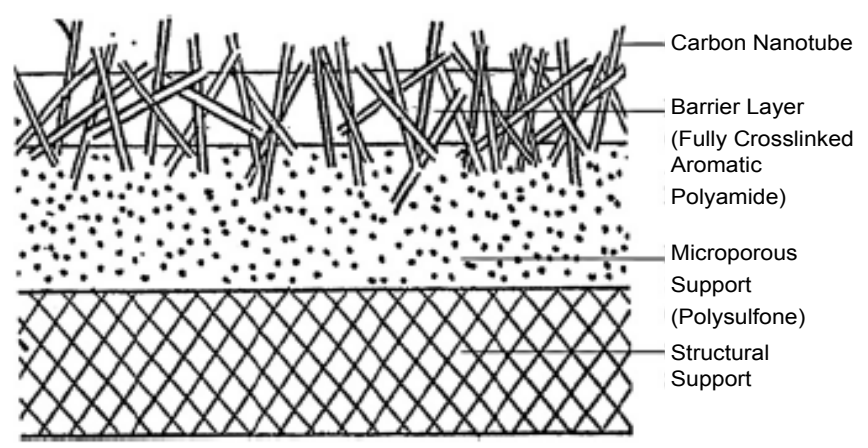

Figure 3: Schematic cross-section of CNTs embedded TFC membrane [1].

need to be functionalized to obtain better solubility in organic solvents. The resulting membrane can be easily adapted into current filtration and RO systems, with the CNTs used being $0.8 \mathrm{~nm}$ in diameter.

Lee et al. [1] exposed the results of a test disclosed in the patent which compares membranes fabricated with and without embedded CNTs, to demonstrate the enhanced flow generated by the CNT pathways. From these results, they concluded that with CNTs present a slightly higher salt rejection was achieved (97.69\% as compared with $96.19 \%)$ and a near doubling of water flux ( $44 \mathrm{l} \mathrm{m}^{-2}$ day $^{-1}$ bar $^{-1}$ as compared with 26 $1 \mathrm{~m}^{-2}$ day $^{-1}$ bar $^{-1}$ ) was obtained. However as the membrane disc that was synthesized was only $47 \mathrm{~mm}$ in diameter, studies on much larger surface area membranes are needed before large scale manufacturing methods can be developed.

\section{Conclusions}

Efficiency of a membrane depends on two parameters: permeate flux and salt rejection. It has been proposed various RO novel membrane materials, but the development of such membranes is only in its initial stages. However, some results from experimental assays show how their efficiency has been improved. Thus, in the first experimental attempt with inorganic membranes (zeolites), these membranes rejected $76 \%$ of $\mathrm{Na}^{+}$ions while permitting a water flux of $0.112 \mathrm{~kg} \mathrm{~m}^{-2} \mathrm{~h}^{-1}(\sim 0.11 \mathrm{l}$ $\mathrm{m}^{-2} \mathrm{~h}^{-1}$ ) (under an applied pressure of $2.07 \mathrm{MPa}$ (20.7 bar) and with $0.1 \mathrm{M} \mathrm{NaCl}$ feed water). In order to improve both parameters, zeolite structure was modified by optimizing $\mathrm{Si} / \mathrm{Al}$ ratio (50/50) and defects in the crystal structure have been minimized. Recently it has been reported that reduction of the thickness of the membrane to $0.7 \mu \mathrm{m}$, providing excellent organic ( $>99 \%)$ and salt rejection $(97.3 \%)$ as well as nearly 4 times improvement in water flux $[1,13,14]$.

Regarding to CNT membranes, as it has been exposed before, fabrication of them is also challenging. Advances in synthesis techniques have opened the possibility of using such CNT membranes for RO due to the discovery of high water fluxes through CNT membranes $[3,16,24,25]$.

Hinds's research group, that introduced the first prototype for a VA-CNT, found that water flow rates increased 4- to 5-fold over those of conventional fluid flow, but they did not report ion selectivity [32].

As it has been explained, the performance of a CNT membrane can be mainly gauged by water flux and salt rejection efficiency, and the smooth and hydrophobic wall of nanotubes facilitates the rapid and frictionless movement of water molecules in chains. Thus, one of the main fields of research is the developing of narrower inner diameter of nanotubes, to get faster transport of water molecules. Furthermore, another point of interest is to research the functionalization of the end tips of nanotubes, in order to change hydrophobicity of the CNT membrane and eventually enhance ion selectivity [17].

Suk and Aluru [41] compared the result from water transport through a porous graphene membrane with water transport through thin carbon nanotube $(\mathrm{CNT})$ membranes. They demonstrated that for larger diameter membranes, water flux was higher through the graphene membrane than of the CNT membrane, while for smaller diameter pores, water flux was lower through the graphene. Moreover, Cohen Tanugi and Grossman [38] showed that nanometer-scale pores in single-layer freestanding graphene can effectively filter $\mathrm{NaCl}$ salt from water.

In the case of Mixed Matrix Membranes, with respect to Nanoparticle/polymeric membranes, the presence of various zeolites in $\mathrm{RO}$ membranes causes changes in membrane characteristics; even they may change polyamide film structure according to some authors. As a result, in almost all of the trials the MMM membrane exhibited and a slight improvement in water flux and in salt rejection relative to the hand cast TFC membrane without zeolite nano-particles [1,42-45].

On the other hand, in the case of carbon nano-tube/polymeric membranes, a study patented by Ratto et al. [48] compared membranes fabricated with and without embedded CNTs. The results demonstrated the enhanced flow generated by the CNT pathways, a near doubling of water flux (44 $\mathrm{l} \mathrm{m}^{-2} \mathrm{day}^{-1} \mathrm{bar}^{-1}$ as compared with $261 \mathrm{~m}^{-2} \mathrm{day}^{-1}$ bar $\left.^{-1}\right)$, with a slightly higher salt rejection $(97.69 \%$ as compared with $96.19 \%)$. However, there is a need for studies on much larger surface area membranes.

\section{Acknowledgement}

This research has been supported by a Grant from the Ministerio de Ciencia e Innovación. Subprograma INNPACTO 2011 (IPT-2011-0778-920000).

\section{References}

1. Lee KP, Arnot, TC, Mattia D (2011) A Review of reverse osmosis membrane materials for desalination - Development to date and future potential. Journal of Membrane Science 370: 1-22.

2. Li L, Lee R (2009) Purification of produced water by ceramic membranes: material screening, process design and economics. Separation and Purification Technology 44: 3455-3484.

3. Humplik T, Lee J, O'Hern SC, Fellman BA, Baig MA, et al. (2011) Nanostructured materials for water desalination. Nanotechnology 22: 1-19.

4. Duke MC, O'Brien-Abraham J, Milne N, Zhu B, Lin JYS, et al. (2009) Seawate desalination performance of Mfi type membranes made by secondary growth. Separation and Purification Technology 68: 343-350.

5. Li L, Dong J, Nenoff TM, Lee R (2004) Reverse osmosis of ionic aqueous solutions on a MFI zeolite membrane. Desalination 170: 309-316.

6. Lin J, Murad S (2001) A computer simulation study of the separation of aqueous solutions using thin zeolite membranes. Molecular Physics: An International Journal at the Interface between Chemistry and Physics 99: 1175-1181.

7. Virkutyte J, Rajender SV, Jegatheesan V (2010) Treatment of Micropollutants in Water and Wastewater. (1stedn), IWA Publishing, London, UK.

8. Li L, Dong J, Nenoff TM, Lee R (2004) Desalination by reverse osmosis using MFI zeolite membranes. Journal of Membrane Science 243: 401-404.

9. Li L, Liu N, McPherson B, Lee R (2008) Influence of counter ions on the reverse osmosis through MFI zeolite membranes: implications for produced water desalination. Desalination 228: 217-225.

10. Dong JH, Li L, Nenoff TM (2008) MFI Zeolite Membranes for Reverse Osmosis Desalination: Effect and Modification of Intercrystalline Pores. In: Desalination Research Progress. Nova Science Publishers Inc, New York; 1-16.

11. Li D, Wang H (2010) Recent developments in reverse osmosis desalination membranes. Journal of Materials Chemistry 20: 4551-4566. 
Citation: Rodríguez-Calvo A, Silva-Castro GA, Osorio F, González-López J, Calvo C (2014) Novel Membrane Materials for Reverse Osmosis Desalination. Hydrol Current Res 5: 167. doi:10.4172/2157-7587.1000167

12. Li L, Liu N, McPherson B, Lee R (2007) Enhanced water permeation of reverse osmosis through Mfi-type zeolite membranes with high aluminum contents. Industrial and Engineering Chemistry Research 46: 1584-1589.

13. Liu N, Li L, McPherson B, Lee R (2008) Removal of organics from produced water by reverse osmosis using Mfi-type zeolite membranes. Journal of Membrance Science 325: 357-361.

14. Lu J, Liu N, Li L, Lee R (2010) Organic fouling and regeneration of zeolite membrane in wastewater treatment. Separation and Purification Technology 72: 203-207.

15. Li L, Dong JH, Nenoff TM (2007) Transport of water and alkali metal ions through MFI zeolite membranes during reverse osmosis. Separation and Purification Technology 53: 42-48.

16. Majumder M, Chopra M, Andrews R, Hinds BJ (2005) Nanoscale hydrodynamics: Enhanced flow in carbon nanotubes. Nature 438: 44.

17. Ahn CH, Baek Y, Lee C, Kim SO, Kim S, et al. (2012) Carbon nanotube-based membranes: Fabrication and application to desalination. Journal of Industrial and Engineering Chemistry 18: 1551-1559.

18. Majumder M, Ajayan PM (2010) Carbon nanotube membranes: A new frontier in membrane science, In: Comprehensive Membrane Science and Engineering.

19. Ajayan PM (1999) Nanotubes from carbon. Chemical Reviews 99: 1787-1800.

20. Mauter MS, Elimelech M (2008) Environmental applications of carbon-based nanomaterials. Environmental Science and Technology 42: 5843-5859.

21. Bianco A (2013) Carbon nanotubes. Organic nanomaterials and delivery research program. Immunopathology and Medicinal Chemistry CNRSUPR3572 Research Group. Institute of Molecular and Cell Biology, University of Strasbourg, France.

22. Corry B (2008) Designing carbon nanotube membranes for efficient water desalination. Journal of Physical Chemistry B 112: 1427-1434.

23. Arjmandi N, Sasanpour P, Rashidian B (2009) CVD synthesis of small-diameter single-walled carbon nanotubes on silicon. Scienta Iranica Transactions D: Computer Science \& Engineering and Electrical Engineering 16: 61-64.

24. Holt JK, Park HG, Wang Y, Stadermann M, Artyukhin AB, et al. (2006) Fas mass transport through Sub-2-nanometer carbon nanotubes. Science 312: 1034-1037.

25. Fornasiero F, Park HG, Holt JK, Stadermann M, Grigoropoulos CP, et al. (2008) Ion exclusion by sub-2 $\mathrm{nm}$ carbon nanotube pores. Proceedings of the National Academy of Sciences of the United States of America 105 (45): 17250 -17255.

26. Lee DH, Lee WJ, Kim SO (2009) Vertical single-walled carbon nanotube arrays via block copolymer lithography. Chemistry of Materials 21: $1368-1374$.

27. Kukovitsky EF, L'vov SG, Sainov NA, Shustov VA, Chernozatonskii LA, (2002) Correlation between metal catalyst particle size and carbon nanotube growth. Chemical Physics Letters 355: 497-503.

28. Joseph S, Aluru NR (2008) Why are carbon nanotubes fast transporters of water? Nano Letters 8: 452-458.

29. Hummer G, Rasaiah JC, Noworyta JP (2001) Water conduction through the hydrophobic channel of a carbon nanotube. Nature 414: 188-190.

30. Kolesnikov A, Zanotti JM, Loong CK, Thiyagarajan P (2004) Anomalously soft dynamics of water in a nanotube: a revelation of nanoscale confinement. Physical Review Letters.

31. Falk K, Sedlmeier F, Joly L, Netz R, Bocquet L (2010) Molecular origin of fast water transport in carbon nanotube membranes: superlubricity versus curvature dependent friction. Nano Letters 10: 4067-4073.
32. Hinds B, Chopra N, Rantell T, Andrews R, Gavalas V, et al. (2004) Aligned multiwalled carbon nanotube membranes. Science 303: 62-65.

33. Kang S, Pinault M, Pfefferle LD, Elimelech M (2007) Single-Walled carbon nanotubes exhibit strong antimicrobial activity. Langmuir 23: 8670-8673.

34. Rodrigues DF, Elimelech M (2010) Toxic effects of single-walled carbon nanotubes in the development of E. coli Biofilm. Environmental Science and Technology 44: 4583-4589.

35. Chiang IW, Brinson BE, Smalley RE, Margrave JL, Hauge RH (2001) Purification and characterization of single-wall carbon nanotubes. Journal of Physical Chemistry B 105: 1157-1161.

36. Lee C, Wei X, Kysar JW, Hone J (2008) Measurement of the elastic properties and intrinsic strength of monolayer grapheme. Science 321: 385-388.

37. Bunch JS, Verbridge SS, Alden JS, van der Zande AM, Parpia JM, et al. (2008) Impermeable atomic membranes from graphene sheets. Nano Letters 8: 24582462.

38. Cohen-Tanugi D, Grossman JC (2012) Water desalination across nanoporous grapheme. Nano Letters 12: 3602-3608.

39. Bae S, Kim H, Lee Y, Xu X, Park JS, et al. (2010) Roll-to-roll production of 30 inch graphene films for transparent electrodes. Nature Nanotechnology 5: 574-578.

40. Sint K, Wang B, Kral P (2008) Selective ion passage through functionalized graphene nanopores. Journal of the American Chemical Society 130: 1644816449.

41. Suk ME, Aluru NR (2010) Water transport through ultrathin grapheme. The Journal of Physical Chemistry Letters 1: 1590-1594.

42. Lind ML, Ghosh AK, Jawor A, Huang X, Hou W, et al. (2009) Influence of zeolite crystal size on zeolite-polyamide thin film nanocomposite membranes. Langmuir 25: 10139-10145.

43. Jeong BH, Hoek EMV, Yan Y, Subramani A, Huang X, et al. (2007) Interfacial polymerization of thin film nanocomposites: a new concept for reverse osmosis membranes. Journal of Membrane Science 294: 1-7.

44. Kim SH, Kwak SY, Sohn BH, Park TH (2003) Design of $\mathrm{TiO}_{2}$ nanoparticle self-assembled aromatic polyamide thin-film-composite (TFC) membrane as an approach to solve biofouling problem. Journal of Membrane Science 211: 157-165.

45. Lee HS, Im SJ, Kim JH, Kim HJ, Min JP, et al. (2008) Polyamide thin-film nanofiltration membranes containing $\mathrm{TiO}_{2}$ nanoparticles. Desalination 219: 4856.

46. Choi JH, Jegal J, Kim WN (2006) Fabrication and characterization of multiwalled carbon nanotubes/polymer blend membranes. Journal of Membrane Science 284: 406-415.

47. Quu S, Wu L, Pan X, Zhang L, Chen H, et al. (2009) Preparation and properties of functionalized carbon nanotube/PSF blend ultrafiltration membranes. Journal of Membrane Science 342: 165-172.

48. Ratto TV, Holt JK, Szmodis AW (2009) Membranes with embedded nanotubes for selective permeability. US Patent 7,993,524. 